\title{
The Effects of Non-uniform Distribution of Oxidizer Flow on High- Frequency Combustion Instability
}

\author{
Peng Chen ${ }^{1}$, Wansheng Nie ${ }^{1}$, Kangkang Guo ${ }^{1}$, Xing Sun ${ }^{1}$, Yu Liu ${ }^{1}$, Haiqing Wang ${ }^{1}$ \\ ${ }^{1}$ Space Engineering University, 101416 Beijing, China
}

\begin{abstract}
The numerical calculation of three-dimensional unsteady combustion for the combustion chamber of LOX/kerosene high pressure staged combustion rocket engine was carried out. By changing the offset ratio of oxygen mass flow rate in the edge area of the injector face, computational studies were conducted to investigate the effects of non-uniform distribution of oxidizer flow on combustion instability for a liquid-propellant rocket engine. The calculation results show that the offset ratio of oxygen mass flow rate changes the distribution of heat release in the combustion chamber. Within a certain range of offset ratio, the non-uniform distribution degree of oxidizer flow enhances the coupling between the pressure and heat release. As a result, it leads to an increase in the pressure oscillation amplitude in the combustion chamber. However, if the offset ratio is too large, the oxygen-fuel ratio will be too small in some regions, which will reduce coupling between the pressure and heat release and increase the damping of combustion instability.
\end{abstract}

\section{Introduction}

Almost every rocket engine in the development process encounters the problem of combustion instability. In particular, high-frequency combustion instability could destroy the cooling structure of the combustion chamber, and instantly ablate the chamber wall, resulting in destroying the structure of combustion chamber. High frequency combustion instability in liquid propellant rocket engines is widely believed to result from a coupling of the complex and dynamic processes of injection, atomization, vaporization, mixing, and chemical reaction with a characteristic response of the gas dynamics in the combustion chamber [1]. Understanding and predicting high-frequency combustion instability in liquid-propellant rocket engine continues to pose significant challenges due to the highly complex and nonlinear nature of turbulent combustion processes. The F-1 rocket engine in the United States has experienced multiple combustion instability during the development process. After conducting more than 2,000 full-scale experiments, it has well suppressed combustion instability [2]. But so far, there is no comprehensive model that can accurately predict the degree of combustion instability in certain engines and certain operating conditions [3].

The cost of conducting full-scale engine experiments is high, and due to the high temperature and pressure environment inside the rocket engine, the data measured in the experimental study is limited. Therefore, Computational Fluid Dynamics (CFD) is needed to analyse the flow field and chemical reaction in the rocket engine in more details and obtain the data of the full flow field [4]. Various studies have been conducted using CFD to study combustion instability. Urbano et al. have explored a multiple-injector $\mathrm{LOX} / \mathrm{GH}_{2}$ rocket engine by making use of Large Eddy Simulations (LES) [5]. A single-element $\mathrm{GO}_{2} / \mathrm{GH}_{2}$ shear-coaxial injector was studied by Masquelet et al. [6] And Morgan et al. studied transverse combustion instability by changing injector configurations in a rectangular chamber rocket engine which uses RP-1, ethane and oxygen as propellants [7].

The LOX/kerosene high pressure staged combustion rocket engine has the advantages of high reliability, environmental protection, low cost and high performance. It is of great practical significance to analyse the combustion instability of the LOX/kerosene staged combustion cycle rocket engine. In this paper, computational studies were conducted to investigate the effects of non-uniform distribution of oxygen mass flow on combustion instability for the LOX/kerosene high pressure staged combustion rocket engine.

\section{Calculation model}

A model was established for the combustion chamber of the LOX/kerosene rocket engine. Oxygen is a gas phase and is treated as a continuum. Kerosene is a liquid phase and its spray process uses the Discrete Phase Model (DPM) which based on the Euler-Lagrange approach. The combustion model uses the Finite-Rate/EddyDissipation model with one-step reaction mechanism. The calculation uses the Reynolds-Averaged NavierStokes (RANS) modelling and pressure implicit splitting 
of operator (PISO) algorithm to simulate the unsteady compressible flow under the three-dimensional condition [8].

\subsection{Physical model}

The injector distribution of the injector face is shown in the Figure 1. High-pressure oxygen-enriched gas is injected from the center hole of the gas/liquid coaxial swirling injector and kerosene is injected from the swirl hole on the sidewall. The effects of non-uniform distribution of oxygen mass flow on combustion instability is studied by changing the offset ratio of oxygen mass flow rate.

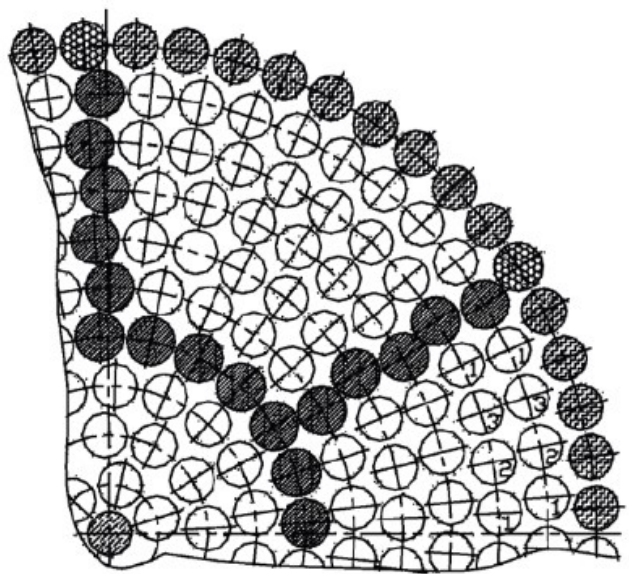

Figure 1. Injector face of rocket engine [9]

\subsection{Gas governing equations}

Numerical calculations are performed in 3D full size. The general format of the control equation in the threedimensional rectangular coordinate system is as follows:

$$
\frac{\partial(\rho \phi)}{\partial t}+\operatorname{div}(\rho \vec{U} \phi)=\operatorname{div}\left[\Gamma_{\phi} \operatorname{grad}(\phi)\right]+S_{\phi}+S_{\phi}^{C}+S_{\phi}^{L}(1)
$$

Where the $\Gamma_{\phi}$ is generalized diffusion coefficient and the is $S_{\phi}$ generalized source item. $S_{\phi}^{C}$ is source item of chemical reaction. $S_{\phi}^{L}$ is source item of liquid phase evaporation. Then the continuity equation, momentum conservation equation and energy conservation equation can be derived from this equation by making $\phi$ and $\Gamma_{\phi}$ take different parameters.

The most widely used RANS equations is currently used. Further, the standard $k-\varepsilon$ turbulence model is Robustness, economy, and reasonable accuracy for a wide range of turbulent flows, so this article uses the standard $k-\varepsilon$ turbulence model.

\subsection{Discrete Phase Model}

The Euler-Lagrange approach with DPM is used where the gas phase is treated as a continuum, while the dispersed liquid phase is solved by tracking a large number of particles through the calculated flow field, i.e. computing the particle trajectories at specified intervals during the fluid phase calculation [10].

\subsection{Finite-Rate/Eddy-Dissipation turbulent combustion model}

The net reaction rate of Finite-Rate/Eddy-Dissipation was controlled by Arrhenius rate and Eddy-Dissipation reaction rate, taking the smaller of the two rates.

\subsection{Chemical Reaction Mechanism}

The chemical reaction uses a one-step reaction mechanism:

$$
\mathrm{C}_{12} \mathrm{H}_{23}+17.75 \mathrm{O}_{2} \rightarrow 12 \mathrm{CO}_{2}+11.5 \mathrm{H}_{2} \mathrm{O}
$$

\subsection{Mesh and boundary conditions}

As shown in the figure 2, the combustion chamber is divided into triangular prism grids with the element number of 510,000. Oxygen is a gas phase, and the DPM model is used for the spray process of kerosene. The spray angle is $40^{\circ}$, and the swirl fraction is 0.5 . The droplet diameter uses the Rosin-Rammler distribution function with an average diameter of $2.5 \mu \mathrm{m}$. The inlet boundary condition is mass-flow-inlet, and the outlet boundary condition is pressure-outlet. The walls are assumed to be adiabatic and are treated as no-slip boundaries.

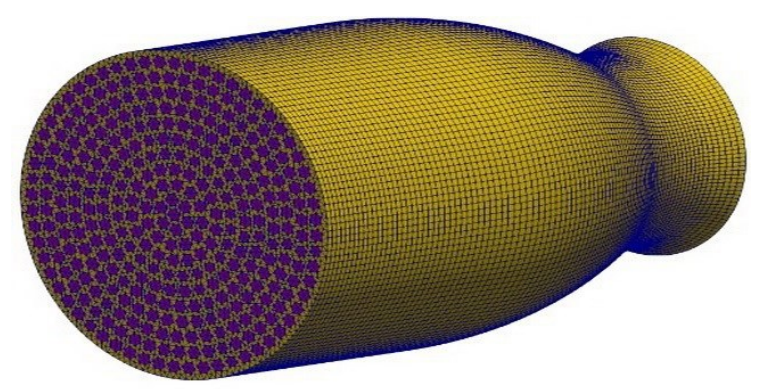

Figure 2. Mesh of the combustion chamber

\section{Calculation}

\subsection{Model validation}

The test pressure of the combustion chamber was $18 \mathrm{MPa}$. And in figure 3, the numerical calculation shows that the mean pressure in the combustion chamber is $17.6 \mathrm{MPa}$ with an error of $2.2 \%$, which validate the reliability of the numerical simulation model built by this paper. 


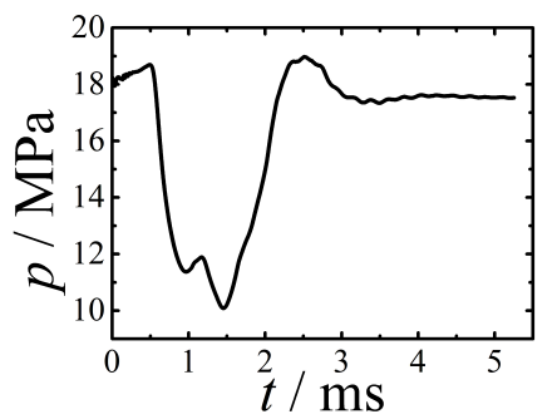

Figure 3. Pressure of the combustion chamber

\subsection{Oxygen mass flow offset}

The oxygen mass flow rate was $371 \mathrm{~kg} / \mathrm{s}$, and the kerosene mass flow rate was $60 \mathrm{~kg} / \mathrm{s}$. As a result, the oxygen-fuel ratio was 6.2. The total mass flow rate of oxygen in the outermost two cycles of the injectors remains stable. It is divided into two half-ring areas A and B. Then set the oxygen mass flow in these areas to a certain proportion to achieve the purpose of flow offset. So case1-case 3 are set up separately, and let the A, B area oxygen mass flow ratio be $3.5: 1,5: 1,8: 1$. In case 0 , mass flow rate of oxygen in outermost two cycles is uniform, which is the reference for other cases. Four monitor points point1-4 are set on the cross-section $10 \mathrm{~mm}$ from the face as shown in figure.

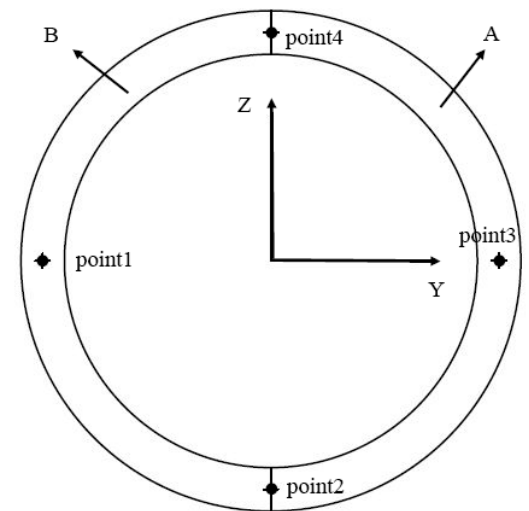

Figure 4. The area divided and the monitor points

\subsection{Results}

The pressure history at the monitor points of case $0-3$ is shown in the figure. It can be seen from the figure that there are pressure oscillations at all monitor points. It is considered that the combustion instability occurs when the pressure oscillation amplitude in the combustion chamber exceeds $10 \%$ of the average pressure [11]. As shown in the Table 1 , the maximum pressure oscillation amplitude of case 1 and case 2 exceeds $20 \%$ of the average pressure, and combustion instability is obvious; while the maximum pressure oscillation amplitude of case 0 is close to $10 \%$, and combustion instability is not obvious; case 3 shows no combustion instability.
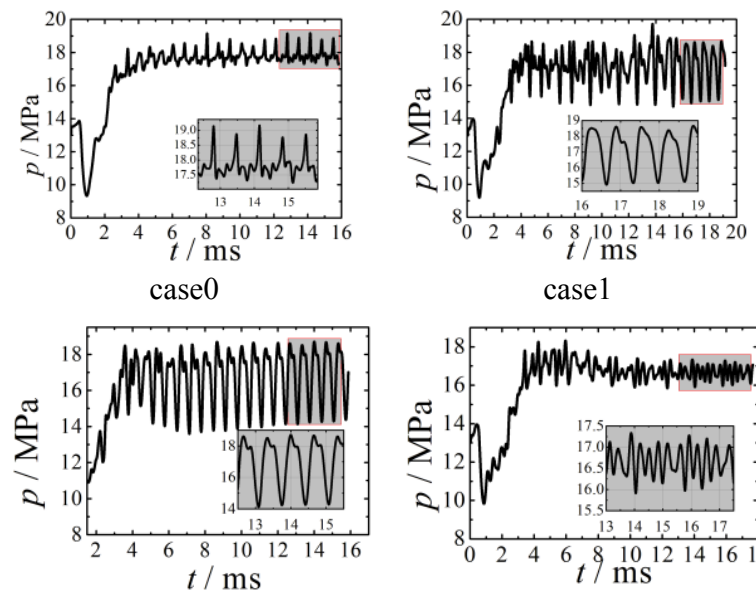

case 2

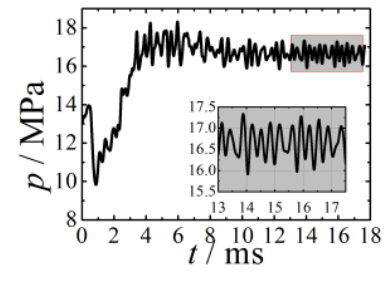

case 3

Figure 5. Pressure of combustion chamber in the four cases

Table 1. the result of the four cases

\begin{tabular}{|c|c|c|c|c|}
\hline & case0 & case1 & case2 & case3 \\
\hline Offset ratio & $1: 1$ & $3.5: 1$ & $5: 1$ & $8: 1$ \\
\hline Mean pressure $/ \mathrm{MPa}$ & 17.8 & 17.3 & 17.2 & 16.9 \\
\hline $\mathrm{p} / \overline{\mathrm{p}}$ & $10.8 \%$ & $22.8 \%$ & $27.2 \%$ & $7.9 \%$ \\
\hline $\begin{array}{c}\text { Oxygen-fuel ratio of } \\
\text { area B }\end{array}$ & 6.2 & 3.4 & 2.5 & 1.9 \\
\hline
\end{tabular}

The pressure frequency of the four monitor points was analyzed using the Fast Fourier Transform (FFT). The dominant frequency of case 1 and case 2 is $1500 \mathrm{~Hz}$, and the frequency is greater than $1000 \mathrm{~Hz}$, indicating that there was high-frequency combustion instability in the combustion chamber; the main frequency of case 0 is $1462 \mathrm{~Hz}$, but the amplitude of main frequency is obviously lower than that of case1 and case2; the main frequency of case 3 is $2867 \mathrm{~Hz}$, and the amplitude is the smallest among the four cases.
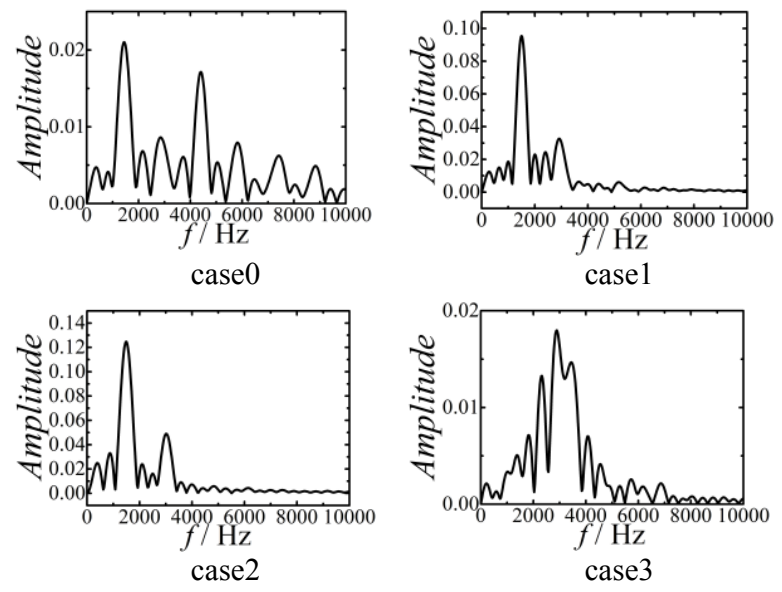

Figure 6. FFT result

The first-order tangential frequency of the combustion chamber is $1661 \mathrm{~Hz}$. And the error with case1 and case2 is about $9.7 \%$. Then the pressure contours of the crosssection $x=10 \mathrm{~mm}$ from the face within one cycle 


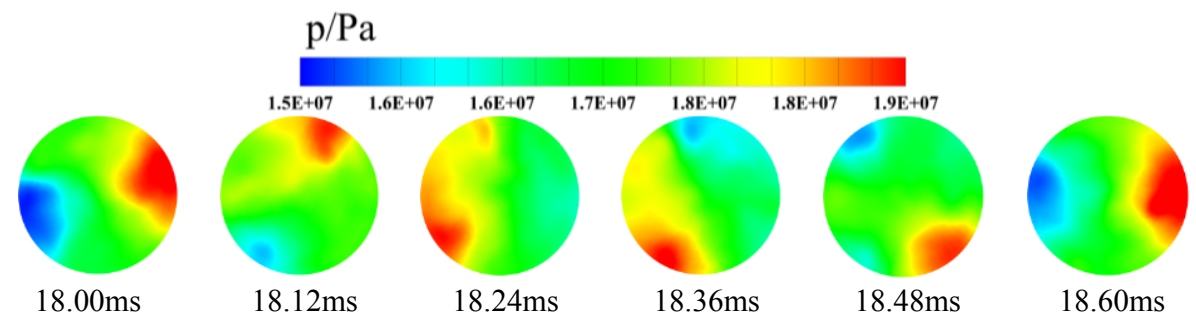

Figure 7. Pressure contours

is shown in the Figure 7, it can be found that there is a rotating high-pressure region and low-pressure region in the combustion chamber, which is the first-order tangential mode of rotation.

\section{Analyse}

Rayleigh criterion is often mentioned when combustion instability is studied [12]. It shows that if heat is added to a pressure oscillation at maximum pressure or if heat is extracted at minimum pressure the pressure oscillation amplitude will increase. In contrast, if the heat is added at minimum pressure or extracted at maximum pressure, the amplitude will decrease $[12,13]$. In the combustion chamber, due to the processes of injection, atomization, vaporization and mixing may have a certain disturbance to the flow field, and the combustion process is also very sensitive to changes in the flow field parameters, there will be a random oscillation of heat release rate [5]. Further, heat release oscillations can cause pressure oscillations. If the heat release oscillation frequency and the pressure oscillation frequency are coupled with the inherent acoustic frequency of the combustion chamber, the pressure oscillation in the combustion chamber will continue to propagate and amplify, eventually causing combustion instability.

The pressure and heat of reaction of point 4 in case 1 and case 2 are shown in figure 8 . It can be clearly seen that when the pressure rises, the heat release rate of the chemical reaction also increases at the same time. Both the frequency and the phase are basically same, indicating that there is a strong coupling relationship between the heat release rate and the pressure.
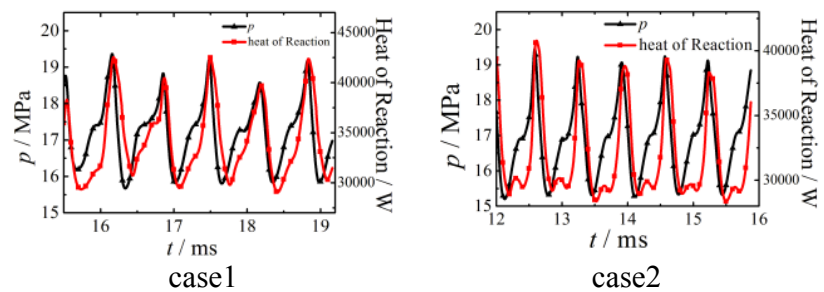

Figure 8. Pressure and heat of reaction in case 1 and case 2

Under different conditions of offset radio, there are different amplitudes of pressure oscillations, indicating that non-uniform distribution of oxygen mass flow has a great influence on the pressure oscillation amplitude of the combustion chamber. In the process of increasing the offset ratio of oxygen from a completely uniform increase to $3.5: 1$ to $5: 1$, the pressure oscillation amplitude in the combustion chamber increased from $10.8 \%$ to $22.8 \%$ to
$27.2 \%$. It can be clearly seen that non-uniform distribution of oxygen mass flow can drive combustion instability.
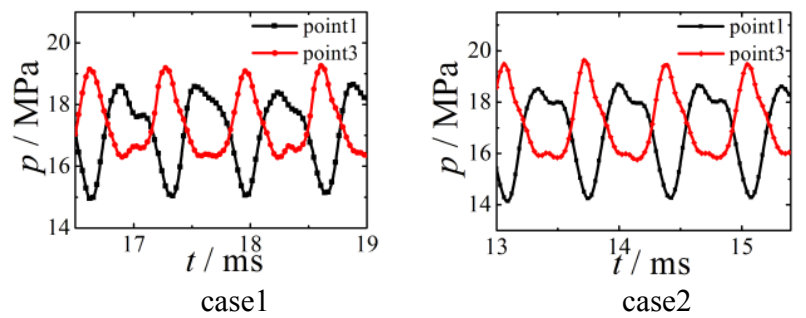

Figure 9. Pressure for point 1 and point 3

As shown in the figure 9, the pressure for point1 and point 3 of case 1 and case 2 are plotted where point 1 is in the $\mathrm{B}$ area and point 3 is in the A area. During the rotation of the high pressure region and the low pressure region in areas $\mathrm{A}$ and $\mathrm{B}$, the pressure peaks at the two monitor points are not consistent. It can be seen that the maximum pressure in the high pressure region increases from area $\mathrm{B}$ to area $\mathrm{A}$, and decreases from area $\mathrm{A}$ to area $\mathrm{B}$. And The minimum pressure in the low pressure region also increases from area B to area A, and decreases from area A to area B. Point 1 and point 3 have the same frequency of pressure oscillation and opposite phases, which indicats that when the high pressure region rotates to area A, the low pressure region rotates exactly to area B. Due to the large oxygen-fuel ratio in area $A$, kerosene complete combustion results in greater heat release. In contrast, the oxygen-fuel ratio in area B is relatively small, so that kerosene is not completely combusted resulting in a small heat release radio, as shown in Figure 10. In this situation, the degree of coupling between the non-uniform heat release and the pressure is further aggravated. According to the Rayleigh criterion, the coupling drives the combustion instability. On the other hand, when the high pressure region rotates to area $\mathrm{B}$ and the low pressure region rotates to area $\mathrm{A}$, the maximum pressure in the high pressure region has a certain attenuation, and the minimum pressure in the low pressure region has a certain increase. However, they can always maintain a certain level until they enter the next cycle, so that pressure oscillations can be maintained and developed.

Finally, due to the dissipation of the wall and other factors in the combustion chamber, a pressure oscillation cycle is formed when the dissipation effect and the coupling of pressure and heat release are balanced. Within this offset radio range, the greater the offset ratio, the stronger the effect of heat release on the pressure oscillation and the larger the pressure oscillation is. 


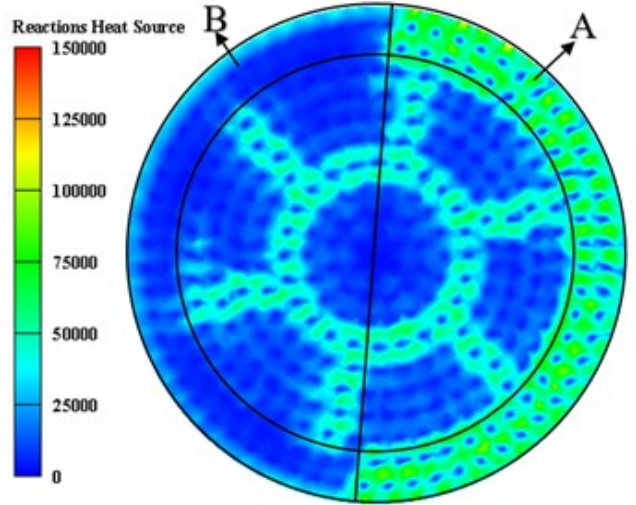

Figure 10. Heat release cloud

As the offset ratio increased from $5: 1$ to $8: 1$, the oxygen-fuel ratio of the area B decreased from 2.5:1 to $1.9: 1$, which is far below the oxygen-fuel ratio of $3.4: 1$ for the propellant just to complete combustion. Therefore, the area B is not fully burned, and the heat release is small, which has a great impediment to the pressure oscillation, resulting in no complete pressure oscillation cycle in the combustion chamber, so no combustion instability occurs.

Therefore, there should be an appropriate offset ratio of oxygen mass flow that maximizes amplitude of pressure oscillation.

\section{Conclusion}

In this paper, the effect of non-uniform distribution of oxygen mass flow on combustion instability for a liquid-propellant rocket engine is investigated by changing the offset ratio of oxygen mass flow rate in the edge area of the injector face. Within a certain range, with the increase of the offset ratio of oxygen mass flow, the pressure oscillation peak also increases, which indicates that the non-uniform distribution degree of oxidizer flow enhances the coupling between the pressure and heat release. It can also be seen that there should be an appropriate offset ratio that can maximize the amplitude of pressure oscillation when combustion instability occurs in the combustion chamber of $\mathrm{LOX} /$ kerosene rocket engine. In this regard, during the design of the rocket engine, the propellant flow distribution should be arranged properly to reduce the probability of combustion instability during the injection process when the propellant is just injected.

\section{References}

1. K. Miller, J. Sisco, N. Nugent, W. Anderson, Journal of Propulsion and Power, 23(5),1102(2007)

2. J.C. Oefelein,V. Yang, Journal of Propulsion and Power, 9(5), 657(1993)

3. V. Sankaran, M. Harvazinski, W. Anderson, D. Talley, ICCFD,7, 3105(2012)

4. R. Smith, G. Xia, W. Anderson, Combustion Theory and Modelling, 16(2), 341 (2012)

5. A. Urbano, L. Selle, G. Staffelbach, B. Cuenot, T. Schmitt, S. Ducruix, S. Candel, Combustion and Flame, 169,129 (2016)

6. M.Masquelet, S.Menon, Journal of Propulsion and Power,26.5,924(2010)

7. C.Morgan, K.Shipley,W.Anderson, Journal of Propulsion and Power, 31(6), 1696(2015)

8. K. Guo, W. Nie, Y. Liu, H. Cai, ICMAE2017,719 (2017)

9. L. Li, J. Chen, Z. Liu, Missiles and Space Vehicles,313,16 (2011)

10. Kozic, S. Ristic, M. Puharic, B. Katavic, Third Serbian Congress on Theoretical and Applied Mechanics,290 (2011)

11. Y.Ga, H.Zhang, X.Wang, Joural of Engineering Thermophysics,33.5,883,(2012)

12. M.Harvazinski,W.Andersony,C.Merkle, Aiaa/asme/sae/asee Joint Propulsion Conference \& Exhibit,47(2011)

13. L.Rayleigh, The Theory of Sound, 2 (1896) 